\title{
Auditory Nerve Perinodal Dysmyelination in Noise-Induced Hearing Loss
}

\author{
Thomas Tagoe, ${ }^{1}$ Matt Barker, ${ }^{1}$ Andrew Jones, ${ }^{1}$ Natalie Allcock, ${ }^{2}$ and Martine Hamann ${ }^{1}$ \\ ${ }^{1}$ Department of Cell Physiology and Pharmacology, and ${ }^{2}$ Electron Microscopy Facility, University of Leicester, Leicester LE1 9HN, United Kingdom
}

\begin{abstract}
Exposure to loud sound (acoustic overexposure; AOE) induces hearing loss and damages cellular structures at multiple locations in the auditory pathway. Whether AOE can also induce changes in myelin sheaths of the auditory nerve (AN) is an important issue particularly because these changes can be responsible for impaired action potential propagation along the AN. Here we investigate the effects of AOE on morphological and electrophysiological features of the centrally directed part of the rat AN projecting from the cochlear spiral ganglion to brainstem cochlear nuclei. Using electron microscopy and immunocytochemistry, we show that AOE elongates the AN nodes of Ranvier and triggers notable perinodal morphological changes. Compound action potential recordings of the AN coupled to biophysical modeling demonstrated that these nodal and perinodal structural changes were associated with decreased conduction velocity and conduction block. Furthermore, AOE decreased the number of release sites in the cochlear nuclei associated with the reduced amplitudes of EPSCs evoked by AN stimulation. In conclusion, AN dysmyelination may be of fundamental importance in auditory impairment following exposure to loud sound.
\end{abstract}

Key words: central auditory pathway; deafness; dorsal cochlear nucleus; dysmyelination; hearing loss; myelin

\section{Introduction}

Myelination of axons provides a means to regulate the conduction velocity essential for sensory processing and higher integrative functions (Huxley and Stampfli, 1949; Waxman, 1980; Kimura and Itami, 2009; Seidl, 2013). The fundamental importance of myelin is highlighted in demyelinating diseases where the speed of conduction along axons is altered and sensory, motor and/or cognitive ability is severely compromised (Nave, 2010). In the auditory system, precise temporal signal transmission is important for sound localization (Oertel, 1999). The auditory nerve (AN) contains mainly myelinated axons projecting from type I spiral ganglion neurons to brainstem cochlear nuclei (Toesca, 1996). The importance of AN dysmyelination is indicated by the occurrence of deafness in patients with Charcot-Marie-Tooth disease, a hereditary disorder caused by genetic mutations related to peripheral nerve myelin (Starr et al., 2003). In rodent models, increased latency of wave I of the auditory brainstem response (ABR) has been linked to mutations affecting myelin (Starr et al., 2003; Jyothi et al., 2010). Moreover, doxorubicin, a demyelinating agent, abolishes the ABR within $24 \mathrm{~h}$ of its injection within the auditory canal (El-Badry et al., 2007). Hearing loss induced by acoustic overexposure (AOE) is well known to be caused by damage to mul-

\footnotetext{
Received Sept. 17, 2013; revised Jan. 8, 2014; accepted Jan. 14, 2014.

Author contributions: M.H. designed research; T.T. performed research; A.J. and N.A. contributed unpublished reagents/analytic tools; T.T., M.B., and A.J. analyzed data; M.H. wrote the paper.

This work was funded by Action on Hearing Loss. We thank the Biomedical Services of the University of Leicester for their technical help, lan Forsythe for providing antibodies, and Nick Hartell and Paul Glynn for helpful discussions.

Correspondence should be addressed to Dr. Martine Hamann, University of Leicester, Department of Cell Physiology and Pharmacology, Medical Sciences Building, PO Box 138, University Road, Leicester LE1 9HN, UK. E-mail: mh86@le.ac.uk.

DOI:10.1523/JNEUROSCI.3977-13.2014

Copyright $\odot 2014$ the authors $\quad 0270-6474 / 14 / 342684-05 \$ 15.00 / 0$
}

tiple structures within the cochlea (Wang et al., 2002) and in the brainstem (Kim et al., 1997). Recently, we described loss of myelin from $\sim 50 \%$ of rat AN axons innervating basal cochlear inner haircell synapses, 3-4 d after AOE (Pilati et al., 2012b). However, how dysmyelination affects action potential propagation along the AN is still unknown. Here, we conduct morphological and electrophysiological studies on AN fibers projecting from the cochlea to the cochlear nucleus. We found that AOE decreased the AN conduction velocity, triggered conduction block and decreased the myelin thickness of the AN projecting toward the auditory brainstem. AOE also triggered notable morphological changes at AN myelin subdomains, such as nodes, paranodes, and juxtaparanodes (Poliak and Peles, 2003) that were associated with decreased conduction velocity.

Based on a recent study showing how myelin loss affected the waveform and temporal precision of action potentials in auditory CNS nerve terminals (Kim et al., 2013), we predicted that AOE would affect presynaptic release at AN terminals. By stimulating the AN to evoke EPSCs in principal cells of the dorsal cochlear nucleus (DCN) in the auditory brainstem, we showed that AOE decreased the amplitude of the EPSCs and confirmed that this was associated with a twofold decrease in the number of release sites onto a given principal cell.

Our findings help to explain how AN dysmyelination can lead to hearing disabilities due to deficits in the action potential propagation and the synaptic transmission in the auditory pathway (Zeng et al., 2005).

\section{Materials and Methods}

Forty-three Wistar rats (male and female) were used. Experiments were performed in accordance with the UK Animals (Scientific Procedures) Act of 1986 Home Office regulations and approved by the Home Office and Leicester University Ethical Committee (PIL 80/8158, PPL 60/4351). 
a
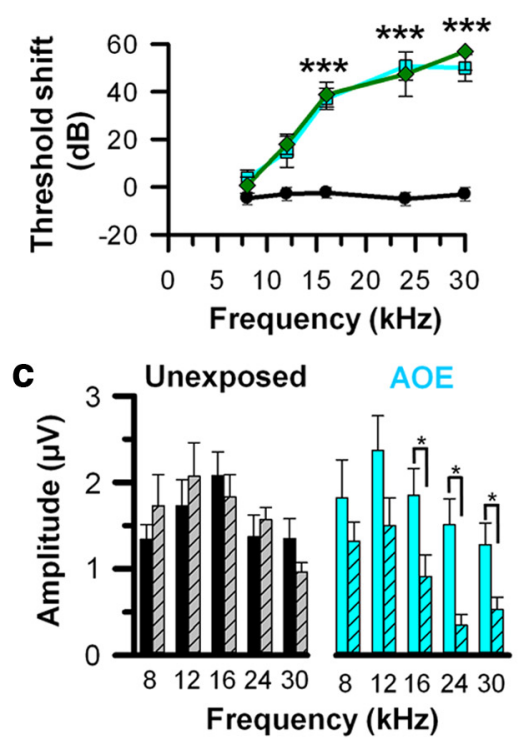

e $\bullet$ Unexposed $\square \mathrm{AOE}$

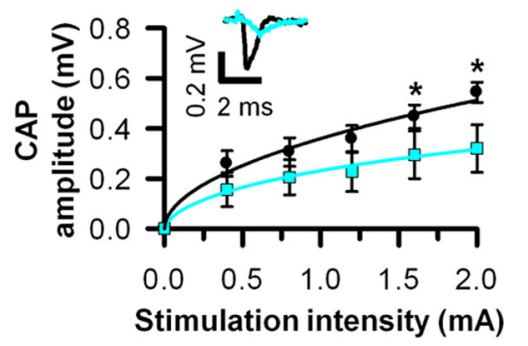

b Unexposed
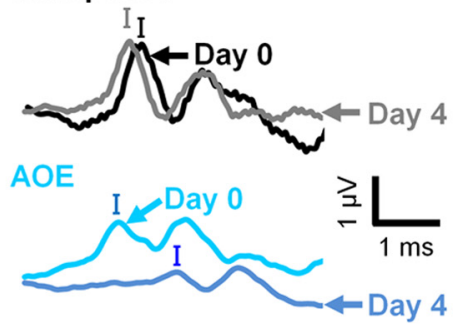

d
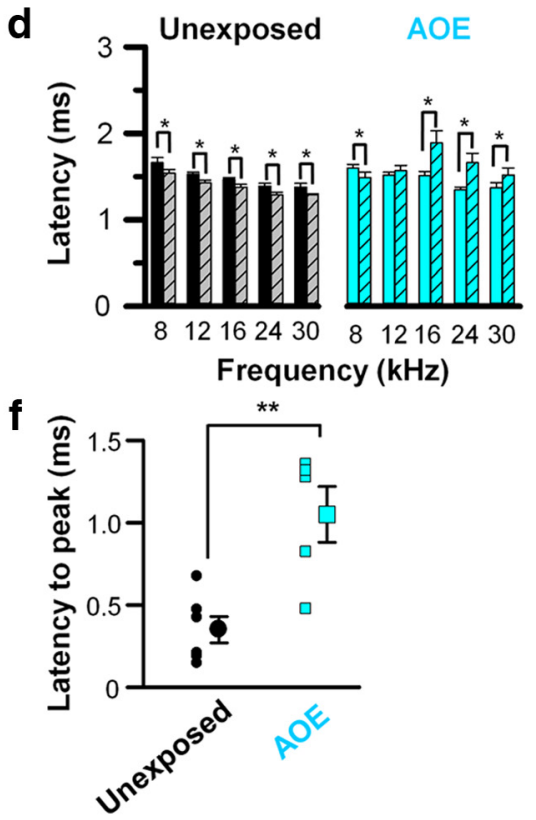

Morphometry. Three Wistar rats per condition were used $4-5 \mathrm{~d}$ after AOE (or anesthesia), between P19 and P24. Transmitted electron microscopy was performed as previously described (Pilati et al., 2012b). The myelin thickness, G-ratio, and number of lamellae of internodal myelin sheaths were analyzed in transverse sections whereas the dimensions of nodes of Ranvier and paranodes were determined on longitudinal sections of centrally directed AN fibers projecting from the cochlea to the brainstem. Nodal regions were selected based on their clear demarcation by paranodal loops on either side. Paranodal regions were selected when continuous loops were observed between the node and the juxtaparanodes. Juxtaparanodes were selected when two adjacent juxtaparanodes originating from a single fiber were located in the same optical plane. To determine the distribution of AN juxtaparanodal marker proteins, immunohistochemistry was performed as previously described (Barker et al., 2012) on 14 coronal brainstem slices per condition (control or AOE), using a mouse monoclonal anti-Kv1.1 antibody (1:100; Neuromab, catalog \#75-007) and a rabbit polyclonal antiCASPR-2 antibody (1:100; Alomone, catalog \#APZ-005). Secondary antibodies were goat antimouse AlexaFluor488 and goat anti-rabbit AlexaFluor568 (1:1000; Life Technologies). Images were obtained using an Olympus Fluoview FV300 confocal microscope. Images from both electron and confocal microscopy were quantified by using ImageJ.

Compound action potential recordings. Coronal brainstem slices $(250 \mu \mathrm{m})$ containing the DCN and the AN (Paxinos and Watson, 2007) were obtained from rats $4-5 \mathrm{~d}$ after $\mathrm{AOE}$ (or anesthesia), between P19 and P24. Dissection of the brainstem and slicing procedures were performed in low $\mathrm{Na}^{+}$medium with 0.1. $\mathrm{mM} \mathrm{Ca}^{2+}$ and $4 \mathrm{~mm} \mathrm{Mg}^{2+}$ (Pilati et al., 2012b). Recordings were performed with a sampling rate of $20 \mathrm{kHz}$, filtering at $4 \mathrm{kHz}$ and using PClamp 9 software (Molecular Devices). Recordings were performed in normal extracellular medium with 2 $\mathrm{mM} \mathrm{Ca}^{2+}$ and $1 \mathrm{mM} \mathrm{Mg}^{2+}$ (at $\left.32-33^{\circ} \mathrm{C}\right)$. Record-

Figure 1. Effects of AOE on ABRs and AN CAPs. $a$, Unlike unexposed (black circles, $n=9$ ), ABR thresholds are increased $4 \mathrm{~d}$ after AOE (blue squares, $n=9$, unpaired $t$ tests) for frequencies $>16 \mathrm{kHz}$, and remain elevated after 4 weeks (green diamonds, $n=5$ ). $\boldsymbol{b}$, Sample ABR traces evoked at $16 \mathrm{kHz}, 94 \mathrm{~dB}$ SPL showing a wave 1 decreased amplitude and prolonged latency (I) $4 \mathrm{~d}$ after AOE. c, d, After $4 \mathrm{~d}$, amplitudes of ABR wave 1 at $16 \mathrm{kHz}, 94 \mathrm{dBSPL}$ are unaltered and latency is decreased ( $n=9$, paired $t$ tests). A0E decreases the amplitude and increases the latency to peak of the ABR wave 1 for frequencies equal or $>16 \mathrm{kHz}(n=9$, paired $t$ tests, solid bars (day 0$)$, stripped bars (day 4)). $\boldsymbol{e}, \mathrm{AOE}$ decreases the maximal amplitude of the CAP along the AN; $n=6$ unexposed; $n=5 \mathrm{AOE} ;$ Mann-Whitney $U$ test. $\boldsymbol{f}$, AOE increases the latency to peak of the AN CAP (evoked at an intensity of $1.6 \mathrm{~mA}$ ). Data are represented as individual points (left) and as means \pm SEM (right); $n=6$ unexposed; $n=5 \mathrm{AOE} ;$ Mann-Whitney U test; ${ }^{*} p<0.05$, ${ }^{* *} p<0.01$, ${ }^{* * *} p<0.001$.

AOE. Rats were aged between P15 and P19 at the first day of AOE, which corresponds to the period after the hearing onset (Geal-Dor et al., 1993). Rats were anesthetized and exposed to loud (110 dB SPL) singletone frequency $(14.8 \mathrm{kHz})$ sound as previously described (Barker et al., 2012; Pilati et al., 2012a). Total exposure time was $9 \mathrm{~h}$ ( $3 \mathrm{~h}$ per day over 3 consecutive days). Age-matched control animals from the same litter were similarly anesthetized but unexposed to AOE. In vitro experiments following the AOE or the anesthesia only were all performed blind.

$A B R$ recordings. Hearing thresholds were determined as the lowest stimulus intensity that evoked reproducible ABR peaks I and II. Hearing threshold shifts were measured as a difference between the hearing threshold measured on day 0 (P15-P19) and the hearing threshold measured $4 \mathrm{~d}$ later, as previously described (Barker et al., 2012; Pilati et al., 2012a). Developmental changes of ABRs occurring during the P15-P19 period (Blatchley et al., 1987) could lead to differential AOE-induced deficits in hearing thresholds. We initially performed the analysis on two separate groups corresponding to an early (P15-P16; $n=9)$ and late $(\mathrm{P} 18-\mathrm{P} 19 ; n=13)$ phase of AOE. Threshold shifts recorded in the early phase group ( $8 \mathrm{kHz}: 2.9 \pm 3.7 \mathrm{~dB} ; 12 \mathrm{kHz}: 16 \pm 6.5 \mathrm{~dB} ; 16 \mathrm{kHz}: 37 \pm 4.6$ dB; $24 \mathrm{kHz}: 51.3 \pm 6.1 \mathrm{~dB} ; 30 \mathrm{kHz}: 51 . \pm 6.8 \mathrm{~dB}$ ) were comparable to threshold shifts recorded in the late phase group ( $8 \mathrm{kHz}: 4 \pm 2.8 \mathrm{~dB} ; 12$ kHz: $12.7 \pm 3.7 \mathrm{~dB} ; 16 \mathrm{kHz}: 36.8 \pm 3.7 \mathrm{~dB} ; 24 \mathrm{kHz}: 50.1 \pm 2.1 \mathrm{~dB} ; 30 \mathrm{kHz}$ : $46.2 \pm 2.9 \mathrm{~dB}$; unpaired $t$ tests). Therefore, all subsequent data were pooled together. ing electrodes were thick-walled glass pipettes (GC150F-7.5 Clark Electromedical, Harvard Apparatus) with a resistance of 3-6 M $\Omega$ filled with the same solution as the extracellular medium. Recordings were performed in the presence of $20 \mu \mathrm{M}$ gabazine (Abcam) and $10 \mu \mathrm{M}$ strychnine (Abcam) to block IPSCs mediated by the activation of GABA-A and glycine receptors respectively. AN fibers were stimulated using a current stimulator (DS2A, Digitimer) delivering $100 \mu \mathrm{s}, 0.2-2 \mathrm{~mA}$ at a rate of $0.3 \mathrm{~Hz}$ to a bipolar electrode (FHC) placed on the AN (Pilati et al., 2012b). Data in Figure $1 e$ were fitted with a Hill function: $y=\mathrm{a} \cdot \mathrm{x}^{\mathrm{b}} /\left(\mathrm{c}^{\mathrm{b}}+\mathrm{x}^{\mathrm{b}}\right)$ where $x$ is the stimulation intensity, $y$ is the compound action potential (CAP) amplitude, $a$ is the maximal response amplitude, $c$ is the value of $x$ at which $y$ reaches halfmaximum, and $b$ is the Hill coefficient. Recording and stimulating electrodes were placed on the AN entering the brainstem as it represents an exceptionally long segment (Guclu et al., 2012). For the conduction velocity, the recording electrode was initially placed close to the stimulating electrode before being moved away in $0.2 \mathrm{~mm}$ steps. Conduction velocity was calculated as distance $\times$ time $^{-1}$ where "distance" represents the distance between the stimulation and the recording electrodes and "time" represents the duration from the artifact of stimulation to the onset of the CAP. Compound action potentials were evoked at $1.6 \mathrm{~mA}$ stimulations. An average of $10 \mathrm{CAP}$ recordings was always used.

Whole-cell recordings. Coronal brainstem slices $(180 \mu \mathrm{m})$ containing the DCN were obtained from Wistar rats used $4 \mathrm{~d}$ after AOE (or anesthe- 
sia) between P19-P23. Using P19-P23 rats allowed us combining morphological with electrophysiological studies notably performing whole-cell patch-clamp recordings to assess effects on synaptic transmission. Wholecell voltage-clamp recordings on DCN fusiform cells were performed as previously described (Pilati et al., 2012a,b). Recordings were performed in the presence of $20 \mu \mathrm{M}$ gabazine and $10 \mu \mathrm{M}$ strychnine. D-AP5 [(2R)-amino-5phosphonovaleric acid] was also used at $50 \mu \mathrm{M}$ to block NMDA receptor mediated currents and allow the isolation of AMPA receptor mediated EPSCs. The patch pipette (3-6 M $\Omega$ resistance) contained the following (in $\mathrm{mM}$ ): 120 $\mathrm{CsCl}, 4 \mathrm{NaCl}, 4 \mathrm{MgCl}_{2}, 0.001 \mathrm{CaCl}_{2}, 10 \mathrm{HEPES}$, 2 Mg-ATP, 0.2 GTP (Tris salt), 10 EGTA, and 2 QX-314 (all from Sigma-Aldrich). Fusiform cells were held at a holding potential of -70 $\mathrm{mV}$. Series resistances of $<12 \mathrm{M} \Omega$ were compensated by $70 \%$. AN stimulation and fitting of the input-output relationship in Figure $3 a$ was performed as above.

Variance-mean analysis. Values for the mean quantal amplitudes $(Q)$, the number of independent release sites $(N)$ and the average probabilities of release $(P)$ were estimated from the relationship between the variance and the mean of the AMPA receptor-mediated EPSC amplitude recorded under different release probability conditions (Clements and Silver, 2000). Sweeps were collected at $0.3 \mathrm{~Hz}$ in the standard extracellular medium and then in solutions containing the following (in $\mathrm{mm}$ ): 0.5 $\mathrm{CaCl}_{2}, 1 \mathrm{CaCl}_{2}, 1.5 \mathrm{CaCl}_{2}, 2.5 \mathrm{CaCl}_{2}$, and 3 $\mathrm{CaCl}_{2}$. Magnesium levels were adjusted to maintain equivalent divalent ion concentrations. The mean EPSC amplitude and variance were calculated over a stable epoch of a minimum of 30-50 events after wash in of each extracellular solution with a different calcium concentration. The variance mean relationship was then fitted with a parabola using the equation: $y=A x-B x^{2}$, where $y$ and $x$ are the EPSC variance and mean amplitude respectively; $A$ and $B$ are free parameters adjusted to optimally fit the parabola. Upon fitting the parabola, $Q$, $P$, and $N$ were calculated using the equations: $Q=A /\left(1+C V^{2}\right)$ and $P=x \cdot(B / A) \cdot\left(1+C V^{2}\right)$ where $C V$ is the coefficient of variation of the EPSC amplitudes at a given calcium concentration calculated as follows: $C V=$ (EPSC standard deviation $\times$ mean amplitude $\left.{ }^{-1}\right)$, and $N=1 \cdot B^{-1}$ (Clements and Silver, 2000).

Mathematical modeling. The model of McIntyre et al. (2002) of a mammalian myelinated axon was adapted from the database archive of the NEURON simulation environment and was used to estimate the effects of the observed changes in the lamella number, the length and the diameter of the nodes, paranodes, and juxtaparanode. Briefly, the axon was modeled as a multicompartmental double cable with nodes of Ranvier, paranodes, and juxtaparanode segments included as separate compartments with different geometry and electrical properties. The axolemma and myelin sheath in paranodal and juxtaparanodal segments each had a passive linear conductance in parallel with the membrane capacitance. Action potentials were generated at the nodes with modified Hodgkin-Huxley equations that incorporate nonlinear fast $\mathrm{Na}^{+}$, persistent $\mathrm{Na}^{+}$, slow $\mathrm{K}^{+}$conductance, a linear leak conductance, and membrane capacitance. The model axons propagated an action potential in response to a suprathreshold, depolarizing current step deliv-
Unexposed

a4
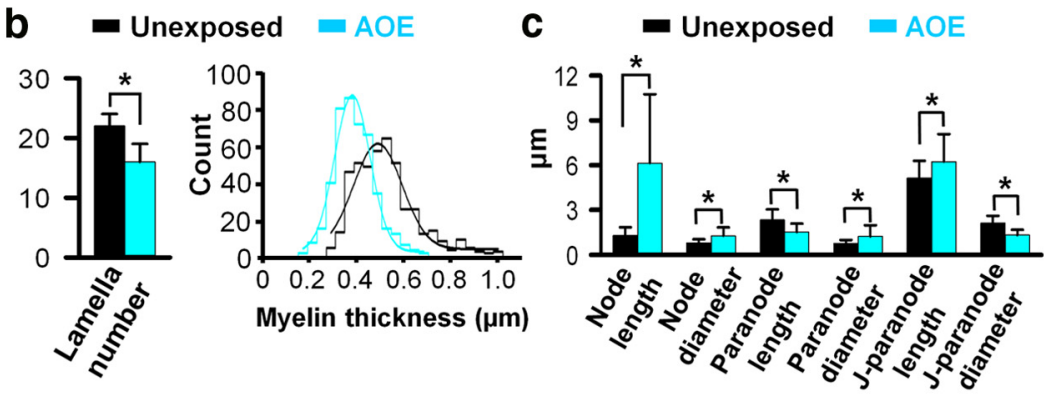

e

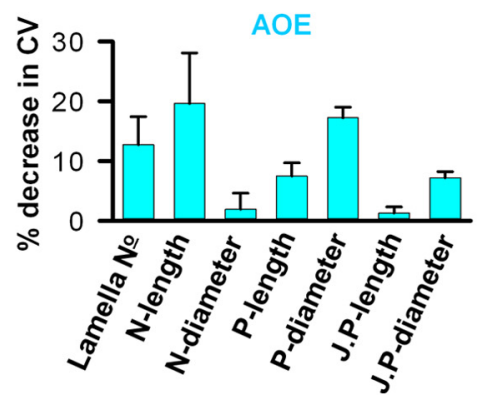

Figure 2. Effects of AOE on morphological and functional properties of the AN. $\boldsymbol{a}$, Electron microscopy showing that AOE decreases the myelin thickness ( $\boldsymbol{a} \mathbf{1}, \boldsymbol{a} 4$, left), decreases the number of lamella wraps ( $\boldsymbol{a} \mathbf{1}, \boldsymbol{a} \mathbf{4}$, right, double-arrowed lines), elongates the node of Ranvier $(\mathbf{a} \mathbf{2}, \mathbf{a 5}$, asterisks between the dotted lines) and increases the diameter of the paranodes ( $\mathbf{a} \mathbf{2}, \mathbf{a 5}$, arrows). Arrows point to the paranodes identified by overlapping loops. a3, a6, Double immunolabeling of Kv1.1 (green) and Caspr2 (red) shows an elongation and a decreased intaparanodes after A0E Scale bars: $a 1$ and $a 4$ left, $1 \mu \mathrm{m} ; a 1$ and $a 4$ right, $100 \mathrm{~nm} ; a 2$ and $a 5,1 \mu \mathrm{m} ; a 3$ and $a 6,2 \mu \mathrm{m} . b$, , AOE decreases the myelin lamella number $(n=62$ each, Mann-Whitney $U$ test). $\boldsymbol{b}$, Right, The Gaussian distribution of myelin exceeding $1 \mathrm{~mm}$. $\boldsymbol{e}$, Modeling data show the contribution of each altered axonal domain property after AOE (c) towards the decreased conduction velocity. Data presented as mean \pm SD $(\boldsymbol{b}, \boldsymbol{c}, \boldsymbol{e})$ or mean \pm SEM $(\boldsymbol{d}) ;{ }^{*} p<0.05$.

ered to the node at one end. Conduction velocity was measured as the distance between the $10^{\text {th }}$ and $20^{\text {th }}$ nodes, divided by the action potential conduction time between those nodes. All simulations were run in NEURON.

Statistical analysis. Data distributions were first tested for normality using D'Agostino and Pearson omnibus normality tests. If distributions were normal, then $t$ tests were used. Alternatively, Mann-Whitney $U$ tests were used when distributions were not normal. For all tests, $p<0.05$ was considered to be statistically significant. Statistics were performed using GraphPad Prism version 5. Unless otherwise specified, data are presented as mean \pm SEM.

\section{Results}

Wistar rats were exposed to a loud sound (AOE: $110 \mathrm{~dB}$ SPL, 14.8 $\mathrm{kHz}, 9 \mathrm{~h}$ ) resulting in an increase in the ABR thresholds $4 \mathrm{~d}$ after 
AOE for frequencies $>16 \mathrm{kHz}$ (Fig. 1a). Hearing threshold elevations after AOE were associated with a decreased wave 1 amplitude and an increased latency (Figs. $1 b-d$ ) indicating an abnormal action potential propagation along the AN. Hearing threshold shifts did not recover 1 month after AOE (Fig. 1a). Abnormal action potential propagation along the $\mathrm{AN}$ was further investigated by field potential recordings of the AN in brainstem slices. AOE reduced the AN CAP amplitude (from $0.54 \pm 0.04$ $\mathrm{mV}, n=6$, to $0.32 \pm 0.07 \mathrm{mV}$ after AOE, $n=5$, at maximal stimulus intensity; Fig. 1e, inset). AOE also increased the CAP latency (from $0.35 \pm 0.08 \mathrm{~ms}, n=6$, to $1.06 \pm 0.19 \mathrm{~ms}$ after $\mathrm{AOE}$, $n=5$; Fig. $1 f$ ). Effects on CAP were in accordance with the decreased amplitude and the prolonged onset of the ABR wave 1 observed in Figure $1 b-d$.

We next tested whether dysfunctional action potential propagation after AOE was due to morphological abnormalities of AN axons. Electron microscopy of transverse sections revealed a decreased number of lamella wraps after AOE (from $23.7 \pm 0.7, n=62$, to $15.8 \pm 0.9, n=62$, after AOE) with a concomitant decrease of the myelin thickness (Fig. 2a1,a4,b). G-ratios were also increased after $\mathrm{AOE}$ (from $0.68 \pm 0.02, n=150$, to $0.72 \pm 0.02$ after AOE, $n=150$; $p<0.05$, unpaired $t$ test). Electron microscopy of longitudinal sections of brainstem containing the $\mathrm{AN}$ was also used to investigate the axonal polarized domains (Poliak and Peles, 2003). We observed notable changes after AOE, particularly a marked elongation of the nodes of Ranvier and a moderate retraction of the paranodes (Fig. $2 a 2, a 5, c)$. By immunolabeling the juxtaparanodal protein Caspr2 and the potassium channel Kv1.1 (Poliak and Peles, 2003), we also revealed that $\mathrm{AOE}$ induced an elongation of the juxtaparanodes (Figs. 2a3,a6,c).

As myelin alterations impair axonal conduction in demyelinating axonopathies (Stephanova and Daskalova, 2008), we next showed that conduction velocity of the AN was decreased from $2.7 \pm 0.3 \mathrm{~m} \cdot \mathrm{s}^{-1}(n=6)$ to $0.9 \pm 0.2 \mathrm{~m} \cdot \mathrm{s}^{-1}(n=7)$ after AOE (unpaired $t$ tests, $p<0.001$; Fig. $2 d$ ). To understand the relationship between the changes in the axonal domains (Fig. $2 c$ ) and the reduced conduction velocity after AOE (Fig. $2 d$ ), action potentials were simulated using a cable model (McIntyre et al., 2002). Mathematical modeling predicted a decreased conduction velocity after AOE due to a decreased thickness of the myelin sheath and altered morphological properties of the axonal subdomains (Fig. 2e).

To obtain further evidence that AOE impairs AN conduction, we used whole-cell recordings to measure EPSCs in DCN fusiform cells evoked by AN stimulation. We showed that the maximal amplitude of EPSCs was decreased after AOE (from $0.51 \pm 0.12 \mathrm{nA}, n=6$, to $0.22 \pm 0.05 \mathrm{nA}$ after AOE, $n=8$, unpaired $t$ test; Fig. $3 a$ ). As AOE also impaired the presynaptic AN volley, we asked whether it reflected on quantal release properties at DCN synapses. Variancemean analyses of the EPSCs showed that the presynaptic effect on the $\mathrm{AN}$ was indeed associated with a twofold decrease in the number of release sites onto a given fusiform cell (from $186 \pm 47, n=6$, to $83 \pm$ $20, n=8$, unpaired $t$ test; Figure $3 b, c)$ in accordance with a decreased activation of AN fibers after AOE.

\section{Discussion}

Damage to multiple cochlear structures: hair cell stereocilia, synapses of inner hair cell-spiral ganglion neurons, supporting cells of the organ of Corti and strial edema can be responsible for AOE-induced noise hearing loss (Wang et al., 2002). These results provide the first evidence for dysmyelination of AN axons contributing to impaired action potential propagation in AOEinduced hearing loss. Increased ABR latencies observed after

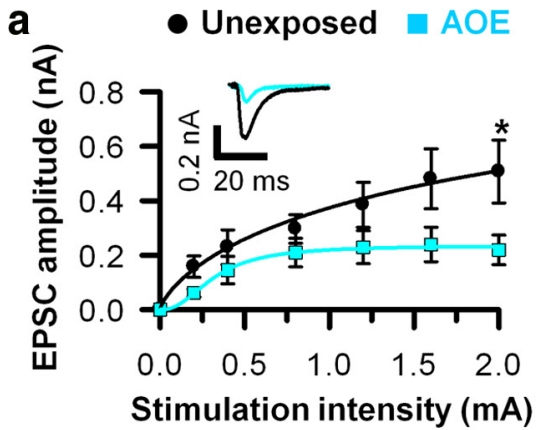

b

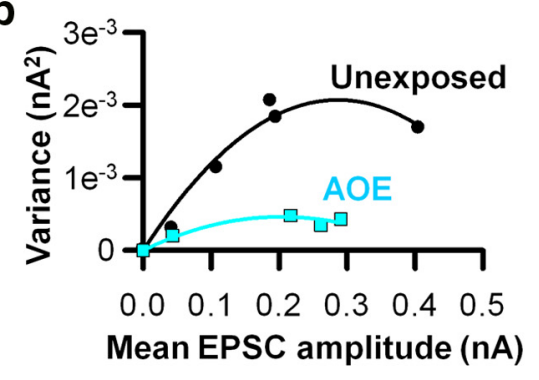

C

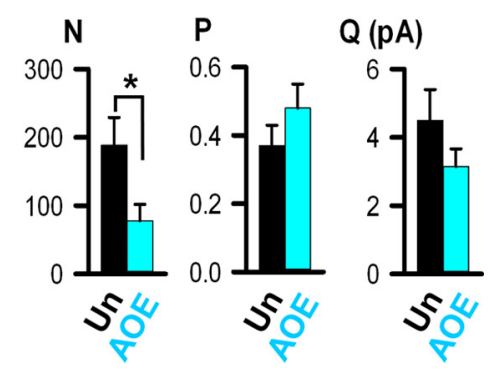

Figure 3. Effects of AOE on EPSCs evoked by AN stimulations. $\boldsymbol{a}, \mathrm{AOE}$ decreases the maximal amplitude of EPSCs recorded in DCN fusiform cells following AN stimulations at $1.6 \mathrm{~mA}$ ( $n=6$ unexposed; $n=8 \mathrm{AOE}$; unpaired $t$ test). Inset, Examples of individual data traces (average of 10 each, black: unexposed; blue: AOE). $\boldsymbol{b}$, Variance-mean relationships for EPSC values in representative fusiform cells are fitted by a parabolic function. $c$, Variance-mean analysis show a decreased number of release sites $(N)$ after AOE whereas the release probability $(P)$ and the quantal size $(Q)$ are unaffected ( $n=6$ unexposed; $n=8 \mathrm{AOE}$, unpaired $t$ tests; ${ }^{*} p<0.05$ ).

AOE could therefore result from dysfunction of the peripheral auditory system and/or action potential propagation along the AN.

We demonstrated AOE-induced reduction in conduction of these AN fibers both by direct electrophysiological measurements and by the resulting decrease in transmission at their central synapses with fusiform cells in the cochlear nucleus. Interestingly, a slowing of conduction velocity of similar magnitude ( $\sim 3$-fold) is evident in abnormally myelinated AN fibers after AOE (Fig. $2 d$ ) and in fibers from the cochlear nuclei to the calyx of Held in Long-Evans shaker (myelin basic protein-deficient) mutant rats (Kim et al., 2013). Cumulatively, ultrastructural changes at and around the nodes appeared to be more significant in reducing the conduction velocity than myelin thinning. This could be due to G-ratios remaining within the $0.6-0.8$ range which is considered an optimal range for axon conduction (Waxman, 1980). The overall time required for saltatory conduction depends largely on the cumulative delays at the nodes because of the time required for discharging nodal capacitance (Huxley and Stampfli, 1949). The elongation of the node (reflecting retraction of paranodal myelin) would increase the nodal capacitance, decrease its membrane resistance and therefore increase the time to reach threshold contributing to slowing the 
conduction velocity (Hartline and Colman, 2007). Deficits of paranodal and juxtaparanodal structures would also result in axonal domains becoming less demarcated, affecting the distribution of axolemmal ion channels (Peles and Salzer, 2000).

Thus, AOE-induced alteration of myelin at and around the nodes of Ranvier are a likely cause of the deficit in the AN fiber recruitment, the conduction block observed for distances exceeding $1 \mathrm{~mm}$ and could contribute to the abnormal auditory brainstem responses as diffuse expression of strategically placed voltage-gated channels would impair the action potential propagation along the AN (Peles and Salzer, 2000; Hossain et al., 2005). Myelin defects could also limit the distance over which those nerve fibers fire in synchrony (Wang et al., 2008) and alter the temporal precision that is critical for sound encoding (Rutherford et al., 2012). The formation of myelin begins at P10 in the rat with the maximal rate of myelin accumulation occurring at P20 (Downes and Mullins, 2013). This could explain the decreased ABR peak 1 latency observed $4 \mathrm{~d}$ after the initial anesthesia in control rats (Fig. 1d). AOE-induced dysmyelination and its functional consequences reported here could be due to an interruption of the development of myelin occurring at that period. However, AOE-induced morphological deficits in adult rodents (Kujawa and Liberman, 2009) suggests that AOE could be directly disrupting myelin rather than interrupting its formation.

Finally, the question arises whether dysmyelination of AN fibers could be the underlying deficits leading to auditory neuropathy, a condition that manifests as a deficit in temporal processing and auditory perception despite intact hair cells (El-Badry et al., 2007). In particular, AN dysmyelination could underlie poorer perceptual abilities and impaired speech discrimination characteristic of auditory neuropathies (Rance, 2005).

Given the mutual inter dependence of axons and glia (Nave, 2010), this fundamental knowledge could eventually contribute to the prevention of spiral ganglion neuron loss that follows months after AOE (Kujawa and Liberman, 2009) and also identified in presbyacusis (Xing et al., 2012).

\section{References}

Barker M, Solinski HJ, Hashimoto H, Tagoe T, Pilati N, Hamann M (2012) Acoustic overexposure increases the expression of VGLUT-2-mediated projections from the lateral vestibular nucleus to the dorsal cochlear nucleus. PLoS One 7:e35955. CrossRef Medline

Blatchley BJ, Cooper WA, Coleman JR (1987) Development of auditory brainstem response to tone pip stimuli in the rat. Brain Res 429:75-84. Medline

Clements JD, Silver RA (2000) Unveiling synaptic plasticity: a new graphical and analytical approach. Trends Neurosci 23:105-113. CrossRef Medline

Downes N, Mullins P (2013) The development of myelin in the brain of the juvenile rat. Toxicol Pathol. Advance online publication. Retrieved Oct. 15, 2013. doi:10.1177/0192623313503518. CrossRef Medline

El-Badry MM, Ding DL, McFadden SL, Eddins AC (2007) Physiological effects of auditory nerve myelinopathy in chinchillas. Eur J Neurosci 25: 1437-1446. CrossRef Medline

Geal-Dor M, Freeman S, Li G, Sohmer H (1993) Development of hearing in neonatal rats: air and bone conducted ABR thresholds. Hear Res 69:236242. CrossRef Medline

Guclu B, Sindou M, Meyronet D, Streichenberger N, Simon E, Mertens P (2012) Anatomical study of the central myelin portion and transitional zone of the vestibulocochlear nerve. Acta Neurochir (Wien) 154:22772283. CrossRef Medline

Hartline DK, Colman DR (2007) Rapid conduction and the evolution of giant axons and myelinated fibers. Curr Biol 17:R29-R35. CrossRef Medline

Hossain WA, Antic SD, Yang Y, Rasband MN, Morest DK (2005) Where is the spike generator of the cochlear nerve? Voltage-gated sodium channels in the mouse cochlea. J Neurosci 25:6857-6868. CrossRef Medline

Huxley AF, Stampfli R (1949) Evidence for saltatory conduction in peripheral myelinated nerve fibres. J Physiol 108:315-339. Medline

Jyothi V, Li M, Kilpatrick LA, Smythe N, LaRue AC, Zhou D, Schulte BA, Schmiedt RA, Lang H (2010) Unmyelinated auditory type I spiral ganglion neurons in congenic Ly5.1 mice. J Comp Neurol 518:3254-3271. CrossRef Medline

Kim J, Morest DK, Bohne BA (1997) Degeneration of axons in the brainstem of the chinchilla after auditory overstimulation. Hear Res 103:169191. CrossRef Medline

Kim JH, Renden R, von Gersdorff H (2013) Dysmyelination of auditory afferent axons increases the jitter of action potential timing during highfrequency firing. J Neurosci 33:9402-9407. CrossRef Medline

Kimura F, Itami C (2009) Myelination and isochronicity in neural networks. Front Neuroanat 3:12. CrossRef Medline

Kujawa SG, Liberman MC (2009) Adding insult to injury: cochlear nerve degeneration after "temporary" noise-induced hearing loss. J Neurosci 29:14077-14085. CrossRef Medline

McIntyre CC, Richardson AG, Grill WM (2002) Modeling the excitability of mammalian nerve fibers: influence of afterpotentials on the recovery cycle. J Neurophysiol 87:995-1006. Medline

Nave KA (2010) Myelination and support of axonal integrity by glia. Nature 468:244-252. CrossRef Medline

Oertel D (1999) The role of timing in the brain stem auditory nuclei of vertebrates. Annu Rev Physiol 61:497-519. CrossRef Medline

Paxinos G, Watson C (2007) The rat brain in stereotaxic coordinates, Ed 6. London: Academic.

Peles E, Salzer JL (2000) Molecular domains of myelinated axons. Curr Opin Neurobiol 10:558-565. CrossRef Medline

Pilati N, Large C, Forsythe ID, Hamann M (2012a) Acoustic over-exposure triggers burst firing in dorsal cochlear nucleus fusiform cells. Hear Res 283:98-106. CrossRef Medline

Pilati N, Ison MJ, Barker M, Mulheran M, Large CH, Forsythe ID, Matthias J, Hamann M (2012b) Mechanisms contributing to central excitability changes during hearing loss. Proc Natl Acad Sci U S A 109:8292-8297. CrossRef Medline

Poliak S, Peles E (2003) The local differentiation of myelinated axons at nodes of Ranvier. Nat Rev Neurosci 4:968-980. CrossRef Medline

Rance G (2005) Auditory neuropathy/dys-synchrony and its perceptual consequences. Trends Amplif 9:1-43. CrossRef Medline

Rutherford MA, Chapochnikov NM, Moser T (2012) Spike encoding of neurotransmitter release timing by spiral ganglion neurons of the cochlea. J Neurosci 32:4773-4789. CrossRef Medline

Seidl AH (2013) Regulation of conduction time along axons. Neuroscience. Advance online publication. Retrieved June 29, 2013. doi:10.1016/j.neuroscience.2013.06.047. CrossRef Medline

Starr A, Michalewski HJ, Zeng FG, Fujikawa-Brooks S, Linthicum F, Kim CS, Winnier D, Keats B (2003) Pathology and physiology of auditory neuropathy with a novel mutation in the MPZ gene (Tyr145->Ser). Brain 126:1604-1619. CrossRef Medline

Stephanova D, Daskalova M (2008) Membrane property abnormalities in simulated cases of mild systematic and severe focal demyelinating neuropathies. Eur Biophys J 37:183-195. CrossRef Medline

Toesca A (1996) Central and peripheral myelin in the rat cochlear and vestibular nerves. Neurosci Lett 221:21-24. CrossRef Medline

Wang SS, Shultz JR, Burish MJ, Harrison KH, Hof PR, Towns LC, Wagers MW, Wyatt KD (2008) Functional trade-offs in white matter axonal scaling. J Neurosci 28:4047-4056. CrossRef Medline

Wang Y, Hirose K, Liberman MC (2002) Dynamics of noise-induced cellular injury and repair in the mouse cochlea. J Assoc Res Otolaryngol 3:248268. CrossRef Medline

Waxman SG (1980) Determinants of conduction velocity in myelinated nerve fibers. Muscle Nerve 3:141-150. CrossRef Medline

Xing Y, Samuvel DJ, Stevens SM, Dubno JR, Schulte BA, Lang H (2012) Age-related changes of myelin basic protein in mouse and human auditory nerve. PLoS One 7:e34500. CrossRef Medline

Zeng FG, Kong YY, Michalewski HJ, Starr A (2005) Perceptual consequences of disrupted auditory nerve activity. J Neurophysiol 93:30503063. CrossRef Medline 